\title{
Literatar. $\left.{ }^{1}\right)$
}

Allatiug, De Purgatorio = Leo Allatius, "De utriusque ecclesiae occidentalis atque orientalis perpetua in dogmate de Purgatorio consensione, Romae 1655.

- De Sym. scr. = Leo Allatius, De Symeonum scriptis diatriba, Pariaiis 1664

Anal. Boll. = Analecta Bollandiana.

$\mathrm{BHG}^{2}=$ Bibliotheca hagiographica graeca edid. socii Bollandiani. Editio altera emendatior, Bruxellis 1909 .

Briquet $=$ C. M. Briquet, Les Filigranes, Dictionnaire historique des marques du papier..., IV tomes, Paris 1907.

Core = Catalogi codicum manuscriptorum bibliothecae Bodleianae pars prima recensionem codicum graecorum continens; confecit O. Coxe, Oxonii 1853.

De Lagarde $=P$. De Lagarde, foannis Euchaitaram quae supersunt; in Abhandl. der histor.-phil. Klasse der Kgl. Gesellschaft der Wisenschaften in Göttingen, Band 28 (1881) $130-137$.

Delehaye, Lég. hagiogr. = Hippolyte Delehaye, Les légendes hagiographiques, 2 Aut., Bruxelle 1906.

- Saints milit. = Hippolyte Delehaye, Les légendes grecques des Saints militaires, Paris 1909.

- Les origines = Hippolyte Delehaye, Les origines du culte des martyrs, Bruxelles 1912.

Deubner, Kosmas und Damian = L. Denbner, Kosmas and Damian. Texte und Einloitang, Leipzig 1907.

Dieterich, Untersuchungen $=\mathrm{K}$. Dieterich, Untersachungen zur Geschichte der griech. Sprache von der hellenist. Zeit bis zum 10. Jahrh. n. Chr. (Byzantinisches Arehiv I), Leipzig 1898.

Dobschütz, Christusbilder $=E$. v. Dobschütz, Christusbilder. Untersuchungen zur chrintlichen Legende. Leipzig 1899. Texte und Untereuchungen zur altchristl. Literatur herausgeg. von 0 . v. Gebhardt und A. Harnack. Nene Folge 3. Band.

Ehrhard, Altchr. Lit. I. = Albert Ehrbard, Die altchristliche Literatar und ihre Erforschung seit 1880. Erster Literaturbericht (1880-1884). Strabburger theolog. Studien. 1. Band, 4. und 5. Heft. - Altchr. Lit. II. = Die altchristl. Literatur und ihre Erforschung von 1884-1900. Freiburg 1900. Strabburger theolog. Studien. Ereter Supplementband.

- Forschungen = Albert Ehrhard, Forschungen zur Hagiographie der griech. Kirche. Römische Quartalschrift 11 (1897) 67-205.

- Griech. Martyr. = Albert Ehrhard, Die griechischen Martyrien (Rede gehalten bei der ersten Jahresversammlung der Wissenschaftl. Gesellschaft in StraBburg am 6. Juli 1907), StraBburg 1907.

Fabricius-Harl., Bibl. Graeca = J. A. Fabricius, Bibliotheca graeca . . . editio quarta curante G. Chr. Harles, Vol. I-XII, Hamburgi 1790-1809. Index, Lipsiae 1838.

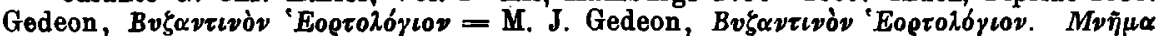

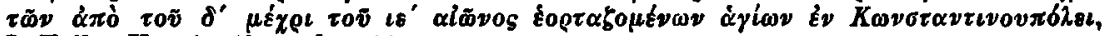
I. Teil. Konstantinopel 1899.

Günter, Legendenstudien $=$ H. Günter, Legendenstudien, Köln 1906.

Harnack = Adolf v. Harnack, Das ursprüngliche Motiv der Abfassung von Märtyrerund Heilungakten in der Kirche. Sitzungaberichte der Kgl. PreuB. Akademie der Wissensch. $1(1910) 106-125$.

Hatzidakis, Einleitung $=$ G. N. Hatzidakis, Einleitung in die neugriechische Grammatik, Leipzig 1892.

Hengstenberg, Drachenkampf $=W$. Hengstenberg, Der Drachenkampî des heiligen Theodoros. Oriens Christianus, Neve Serie 2 (1912) 78-106; 3 (1913) 241-280.

Jannaris = A. N. Jannaris, An historical greek grammar, London 1897.

Kellner, Heortologie = H. Kellner, Heortologie oder die geschichtliche Entwicklung des Kirchenjahres und der Heiligenfeste, 3. Aufl. Freiburg i. B. 1911.

Krumbacher, Theodosius $=K$ K. Krumbacher, Studien zu den Legenden des hl. Theodosius, Sitzungsber. der philos.-philol. und histor. Klasse der Kgl. Bayer. Akademie der Wissensch. 1892, II. Heft S. 217-379, München 1892.

1) Die sonst gelegentlich herangezogenen Werke sind an den betreffenden Stellen genau angeführt. 
- Miszellen z. Romanos =K. Krumbscher, Miszellen zu Romanos, Abhandl. der Kgl. Bayer. Akademie der Wissensch. I. Klasse, Bd. 24, Abt. III S. 1 ff., München 1907.

- GBL $^{2}=$ K. Krumbacher, Geschichte der byzantinischen Literatar, 2. Aufl. München 1897.

- griech. Liter. = K. Krumbacher, Die griechische Literatur des Mittelalterg. Kultur der Gegenwart, I. 8. S. 239-290, 2. Aufl. Leipzig 1907.

Kühner-BlaB | Raphael Kühner, Ausführliche Grammatik der griech. Sprache.

Kühner-Gerth \} I. Teil, Elementar- und Formenlehre, 3. Aufl, bes. von F. BlaB. Hannover 1890 und 1892. II. Teil, Satzlehre, 3. Aufl. bes. von B. Gerth, Hannover und Leipzig 1898 und 1904.

Kyrillos, Vita Euthymii = Kyrillos von Skythopolis, Vita S. Euthymii, in Analecta Graeca der Mauriner tom. I. Pariaiig (1688) p. 1-99.

- Vita Sabae = Kyrillos von Skythopolis, Vita S. Sabae in der Cotelerius-Sammlung: Ecclesiae Graecae monumenta tom. III, Parisiig (1686) p. 220-376.

Lampros = Spyr. P. Lampros, Catalogue of the greek manuscripts on Mount Athos. Vol. I. Cambridge 1895; Vol. II. Cambridge 1900.

Leontios von Neapolis = Leontios von Neapolis, Leben des hl. Johannes des Barmherzigen, Erzbischofe von Alexandrien, herausgeg. von H. Gelzer, Freiburg i. B. 1893.

Lucius, Die Anfänge = Ernst Lucius, Die Anfänge des Heiligenkultus in der christlichen Kirche, herausgeg. von Gustav Anrich, Tübingen 1904.

Marci Diaconi vita Porphyrii = Marci Diaconi vita Porphyrii episcopi Gazensis edid. societatis philologme Bonnensis sodales, Lipsiae 1895.

Meyer, A. = Arnold Meyer, Entstehung und Entwicklung des Weibnachtsfestes, 2. Aufl. Zürich 1913.

Mertel = H. Mertel, Die biographische Form der griech. Heiligenlegenden, München 1909.

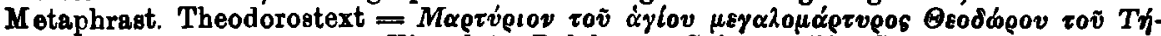
owvos, heransgeg. von Hippolyte Delehaye, Saints milit. S. 136-160 und von Latyø̌ev nach dem Cod. Mosquensis 876 in Menologii anouymi Bjzantini aaec. $\mathbf{X}$ quae supersunt. Fasciculus prior, Februarinm et Martium menses continens. Petropoli 1911.

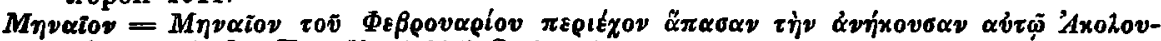
víav, 6. Aufl. Venedig (1895) S. 89-91.

Nuth $=$ A. Nuth, De Marci Diaconi vits Porphyrii episcopi Gazensis quaestiones historicae et grammaticae, Bonnae 1897.

Omont, Invent. = H. Omont, Inventaire sommaire des manuscrits grecs do la Bibliothèque nationale, Paris 1886 - 1888, 3 Bde.; Introduction et la table alphabétique 1898.

Pediasimos $=$ M. Treu, Theodori Pediasimi eiusque amicorum quae exstant. Programm des Viktoria-Gymnasiums zu Potadam S. 17-25: Tov a

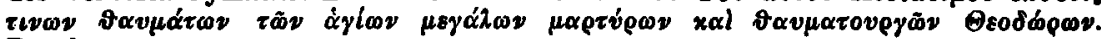
Potsdem 1899.

Reil = Moritz Reil, Zur Akzentuation griechiscber Handachriften. B. Z. XIX (1910) 476-629.

Starck $=$ H. Starck, Theodoros Teron. Textkritiache Ausgabe der vormetaphrastischen Legende, Freising 1912.

Studites = Theodoros Studites, De s. Theodoro Duce, bei Pitra, Analecta sacra, I. Parisiis (1876) 361-366.

Unener, Theodosios $=$ H. Usener, Der hl. Theodosios. Schriften des Theodoros und Kyrillos. Leipzig 1890.

- Weihnachtsfest $=$ H. Usener, Das Weihnachtsfest. Religionsgesch. Untersuchungen. 1. Teil, 2. Aufl, besorgt von H. Lietzmann, Bonn 1911.

- Acta m. Anast. = H. Usener, Acta martyria Anastasii Persae, Bonnae 1894.

Vailhé = Chrysippe, prêtre de Jéruealem. Revue de l'Orient chrétien 12 (1905) 96-99.

- Saint Euthyme =. Vailhé, Saint Euthyme le grand moine de Palestine (376-473). Extrait de la Revue de l'Orient cbrétien, tom. 12-14 (1907-1909), Paris 1909.

Vogeser = J. Vogeser, Zar Sprache der griechischen Heiligenlegenden, München 1907.

Weyh, Kosmas and Damian = Wilhelm Weyh, Die syrische Kosmas- und DamianLegende. Programm des K. human. Gymnas. Schweinfurt für das Schuljahr 1909/10. Sehweinfurt 1910.

- Barbara-Legende = Wilhelm Weyh, Die syrische Barbara-Legende. Programm dea K. human. Gymnasinms Schweinfurt 1911/12. Schweinfurt 1912.

Wulf, Heilige und Heiligenverehrung $=M$. Wulf, Utber Heilige nnd Heiligenverehrung in den ereten chrietlichen Jahrhunderten, Leipzig 1910 